\title{
Rapid Yield Learning Through Optical Defect and Electrical \\ Test Analysis
}

LONF-980225.-

\author{
Shaun S. Gleason \\ Kenneth W. Tobin \\ Thomas P. Karnowski \\ Oak Ridge National Laboratory \\ P.O. Box 2008 \\ Oak Ridge, TN 37831-6011
}

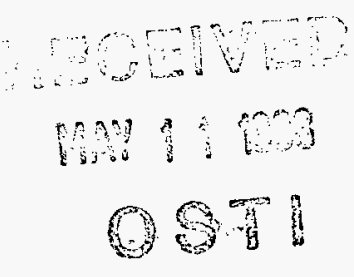

Fred Lakhani

SEMATECH

Austin, TX 78741-6499

"The submitted manuscript has been authored by a contractor of the U.S

Government under contract No. DE-AC05-960R22464. Accordingly, the

U.S. Government retains a nonexclusive, royalty-free license to publish or

reproduce the published form of this contribution, or allow others to do so,

for U.S. Govemment purposes."

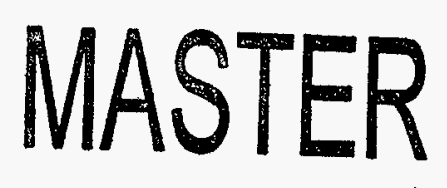

\section{DSSTRIBUTION OF THAS DOCUMENT IS UNLMMTIED

Prepared by OAK RIDGE NATIONAL LABORATORY, Oak Ridge, Tennessee 37831-6285, managed by LOCKHEED MARTIN ENERGY RESEARCH CORP. for the U.S.

DEPARTMENT OF ENERGY under contract DE-AC05-960R22464. 


\section{DISCLAIMER}

This report was prepared as an account of work sponsored by an agency of the United States Government. Neither the United States Government nor any agency thereof, nor any of their employees, make any warranty, express or implied, or assumes any legal liability or responsibility for the accuracy, completeness, or usefulness of any information, apparatus, product, or process disclosed, or represents that its use would not infringe privately owned rights. Reference herein to any specific commercial product, process, or service by trade name, trademark, manufacturer, or otherwise does not necessarily constitute or imply its endorsement, recommendation, or favoring by the United States Government or any agency thereof. The views and opinions of authors expressed herein do not necessarily state or reflect those of the United States Government or any agency thereof. 


\title{
Conf $-980225=$
}

\section{Rapid Yield Learning through Optical Defect and Electrical Test Analysis}

\author{
Shaun S. Gleason ${ }^{a, 1}$, Kenneth W. Tobin ${ }^{a}$, Thomas P. Karnowski ${ }^{a}$, Fred Lakhani ${ }^{b}$ \\ ${ }^{a}$ Oak Ridge National Laboratory ${ }^{2}$, Oak Ridge, TN, 37831 \\ ${ }^{b}$ SEMATECH, Austin, TX, 78741-6499
}

\begin{abstract}
As semiconductor device density and wafer area continue to increase, the volume of in-line and off-line data required to diagnose yield-limiting conditions is growing exponentially. To manage this data in the future, analysis tools will be required that can automatically reduce this data to useful information, e.g., by assisting the engineer in rapid root-cause diagnosis of defect generating mechanisms. In this paper, we describe a technology known as Spatial Signature Analysis (SSA) and its application to both opticallydetected defect data as well as electrical test (e-test) bin data. The results of a validation study are summarized that demonstrate the effectiveness of the SSA approach on optical defect wafermaps through field-testing at three semiconductor manufacturing sites on ASIC, DRAM and SRAM products. This method has been extended to analyze and interpret electrical test data and to provide a pathway for correlation of this data with in-line optical measurements. The image processing-based, fuzzy classifier system used for optical defect SSA has been adopted and applied to e-test binmaps to interpret and rapidly identify characteristic patterns, or "signatures", in the binmap data that are uniquely associated with the manufacturing process. An image of the binmap is created, and features such as mass, simple moments, and invariant moments are extracted and presented to a pair-wise, fuzzy, k-NN classifier. The preliminary performance results show an $84 \%$ correct e-test signature classification rate, even under sub-optimal training conditions.
\end{abstract}

Keywords: electrical test, spatial pattern recognition, semiconductor inspection, moments

\section{INTRODUCTION}

As the semiconductor device density continues to increase and the critical dimensions become smaller, the size of killer defects is shrinking. As manufacturing moves towards the next technology node, $0.18 \mu \mathrm{m}$, these killer defects can be on the order of $0.09 \mu \mathrm{m}$ in size. These defects will be difficult, or even impossible in some cases, to detect using the well-established optical microscope-based defect inspection systems. Although new inspection technologies are under development, the importance of electrical testing of devices will play an increasingly important role in predicting product yield and determining the health of the manufacturing process. For the case of binmap electrical-test (e-test) data, it has been demonstrated that problems in the semiconductor manufacturing process will many times be closely linked with a particular two-dimensional spatial pattern, or signature, of die failing a given bin on the wafer. For example, a collection of electrical failures around the outside edge of the wafer may indicate a spin coater problem. Also, a linearly oriented collection of failed die may be indicative of a mechanical handling scratch imparted on the wafer during processing. If these typical patterns can be automatically identified and linked to a specific manufacturing problem, this will accelerate the yield learning process by helping the fabrication engineer quickly diagnose the problem. This paper describes an image-based approach to automate this process of e-test, specifically binmap, signature analysis. The collection of failed die in each category, or bin, of the binmap data set is converted into a binary image where each failed die represents a "on" pixel in the binmap image. This binary image is subsequently processed to extract features about the spatial distribution of the die. Some of the features that are measured are central moments, coverage, and centroid, for example. These features are then submitted to a user-trainable, fuzzy k-NN classifier for

\footnotetext{
${ }^{1}$ S.S.G. (correspondence): Email: gleasonss@ornl.gov: WWW: http://www-ismv.ic.ornl.gov: Ph: 423-574-8259; Fax: 423-574-6663

${ }^{2}$ Managed by Lockheed Martin Energy Research Corp. for the U.S. Department of Energy under contract DE-AC05-96OR22464.
} 
automatic binmap signature classification. This technique for binmap signature analysis uses as its foundation a technology known as spatial signature analysis (SSA) that has been developed for detection and classification of optical defect spatial signatures. Many of the features used in the binmap signature analysis are also used in the optical SSA technique, and the fuzzy k-NN classifier is identical to that used for optical defect SSA. The binmap signature analysis technique has been tested on over 2000 binmap data files collected from three different semiconductor manufacturers with promising results. These binmap signature results are presented as well as the results of an extensive, 6-month validation study of the optical SSA technology performed at three different semiconductor manufacturing facilities.

\section{SSA Review}

Optical inspection of semiconductor wafers has long been the primary means of investigating the sources of wafer defects. Semiconductor yield engineers use high-resolution images of individual defects collected off-line to assess problems in the manufacturing process. Since high-resolution off-line defect review is time consuming and expensive, process engineers also use low resolution defect wafermaps from in-line optical inspection tools to determine the potential source of problems in the manufacturing process. They accomplish this by analyzing and sourcing unique spatial distributions or "signatures" of defects on the wafer surface. Fig. 1 shows an example of a high-resolution optical defect and a single and stacked wafermap containing various spatial signatures. Even when these spatial signatures do not contain significant portions of killer defects (i.e., defects that result in electrical faults), they do provide a diagnostic window into the manufacturing process. SSA attempts to emulate this process to provide the fabrication engineer with faster time-to-results, a critical requirement for effective yield learning and yield management.

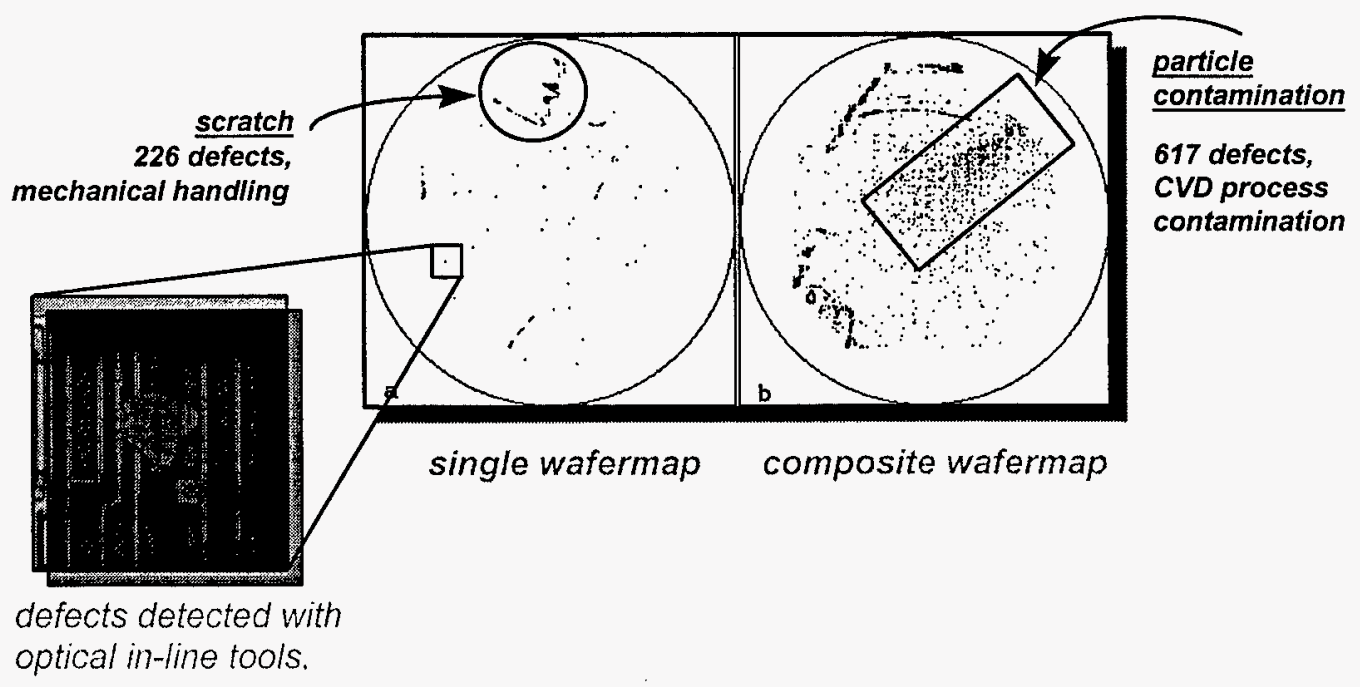

Figure 1. A spatial signature is defined as a unique distribution of wafer defects originating from a single manufacturing problem: (a) single wafer containing scratch signatures. (b) stack of wafers superimposed highlighting a subtle systematic particle contamination problem.

SSA automatically collects defects on a wafermap that come from a single manufacturing source. A usertrained classifier assigns a label that identifies the root problem. SSA begins the signature classification process by converting the electronic wafermap file into a gray-scale image where each pixel is assigned an intensity value according to the number of defects in the subtended area. Each pixel represents a first level clustering of the individual defects. Clusters of pixels, denoted as "objects", in this density image are connected into multi-element objects (e.g., a multi-element scratch) by means of an advanced clustering procedure. Objects are grouped into high-level "sets" depending on their proximity to neighboring clusters and on their morphology ${ }^{1,2}$. 
Once an object has been assigned to a high-level set and characterized, its features are sent to a classifier where a user-defined label is assigned to the result. For this work, a pair-wise fuzzy k-Nearest Neighbor $(\mathrm{kNN})$ approach has been adapted ${ }^{3,4}$ which uses a unique feature reduction procedure to optimize classifier performance ${ }^{5}$. For industrial pattern recognition problems where it is difficult to ascertain a statistical parameterization for the large variety of class types encountered, non-parametric classifiers such as nearest mean or $\mathrm{kNN}^{6}$ apply well. Furthermore, in an industrial setting, it is often required that the classifier system begins to classify new data with few training examples. Bayesian classifiers ${ }^{7}$ and neural networks ${ }^{8}$ can also work well but generally require large sample populations to estimate the appropriate parameters for their method and would therefore be difficult to implement for this application. This is primarily due to the diverse nature of the patterns that arise for different manufacturing processes and processing facilities coupled with the length of time required to collect large sample populations. Also, over the period of time required to collect large sample sets, acceptable process variations can occur which confuse the boundaries between classes. The pair-wise fuzzy kNN classifier training set can readily be maintained over time (e.g., by including and excluding examples based on time and date), and can operate adequately with relatively few examples for each class?

\section{Electrical Test Wafermaps}

As will be described in Section 3, the SSA approach has been adapted to analyze e-test data. Similar to optical defect wafermaps, e-test wafermaps in many cases contain characteristic patterns, or signatures, that give insight into the health of the semiconductor manufacturing process. E-test wafermaps are commonly comprised of either bit data or bin data. Wafermaps created from bit data are called bitmaps, while wafermaps created from bin data are referred to as binmaps. Bit data result from performing electrical tests on the individual bits in a memory device, while bin data describe the results of electrical testing of individual die on a wafer. A bin can be viewed as a "bucket" classification into which all of the die that meet that classification fall. The most intuitive e-test bins are PASS and FAIL. Whether bit data or bin data are used, the e-test wafermap is created for viewing by "mapping" the results of these electrical tests onto a 2-D space.

\section{OPTICAL SSA VALIDATION STUDY}

This section describes the approach and results of a validation study carried out to determine the effectiveness of SSA on optical defect wafermaps. The degree of success experienced during this study was a strong motivation to extend SSA to handle e-test data.

\section{Purpose}

SSA research was initiated between SEMATECH and Oak Ridge in 1995. Initial development of the algorithms for signature segmentation and subsequent classification were based on early discussions with fabrication engineers and a broad spectrum of wafermap files donated to Oak Ridge by the various semiconductor manufacturers. The validation exercise has provided us with the ability to test the maturity of this research and the SSA C++ software library in three different manufacturing environments on three separate products: ASIC, DRAM, and SRAM.

The SSA method is built on two primary technologies: advanced clustering which segments the defects of a given signature into distinct groups; and signature classification which assigns a user-defined class label to each unique group. The ability of SSA to accurately source signatures to manufacturing problems, provide new information for statistical process control, provide improved off-line review-tool performance and throughput, and provide new data for yield analysis, all depend on this base functionality. The validation results that follow are intended to quantify the performance of SSA for advanced clustering and classification performance.

Three sites participated in this validation study and Fig. 2 shows the representative characteristics of the three sites in relation to the well-known yield curve. The yield curve represents various levels of maturity in the manufacturing life cycle of a semiconductor device/product. 
SSA technology has application to most phases of process development and product manufacturing. The left-most region of the curve is representative of the exploratory R\&D phase. In this phase of process development, there are very few devices manufactured and yield is not the driving issue. At this stage, very little automation is required for data analysis. As the exploratory phase moves into the process development phase, higher wafer volumes necessitate the initiation of automation procedures to monitor a significant fraction of product. During the process development stage, systematic events are the dominant source of concern and most prevalent. Systematic events include mechanical wafer handling damage, plasma etch damage from arcing, systematic particle contamination during chemical vapor deposition, spinon material contamination (e.g., glass, resist), and many other possible process related scenarios.

As the process development cycle begins to mature, it progresses to the yield learning phase. It is during this phase that the yield engineer will attempt to determine root-cause sources of systematic events and quickly eliminate them to increase yield. During this phase the number of wafers processed increases dramatically and SSA has the potential to very quickly assist the fabrication engineer in locating and correcting a large variety of problems in the manufacturing process.

The yield learning ramp is of critical importance to the manufacturer. The rate at which yield learning is achieved is critical to the realization of a return on investment. By analyzing the maximum number of wafermaps with SSA during this stage, it is anticipated that yield learning can proceed much more quickly and that yield-impacting events can be more efficiently localized and corrected.

As the process matures over time, it enters the yield-monitoring phase. At this point the process technology is mature and acceptable device yields are maintained. A very small percentage of yield loss is related to systematic events for the controlled process and yield is primarily limited by random events, e.g., particles generated within process tools, or those originating from process gases and chemicals. At this phase, wafer volume is high and systematic yield loss is hard to detect. While SSA provides its main analysis advantage by monitoring single wafers during the yield learning phase, it will provide continued automatic capabilities during the yield monitoring phase by detecting subtle systematic events by overlaying multiple wafers together over single or multiple lots. It can also be used to monitor population statistics of random events.

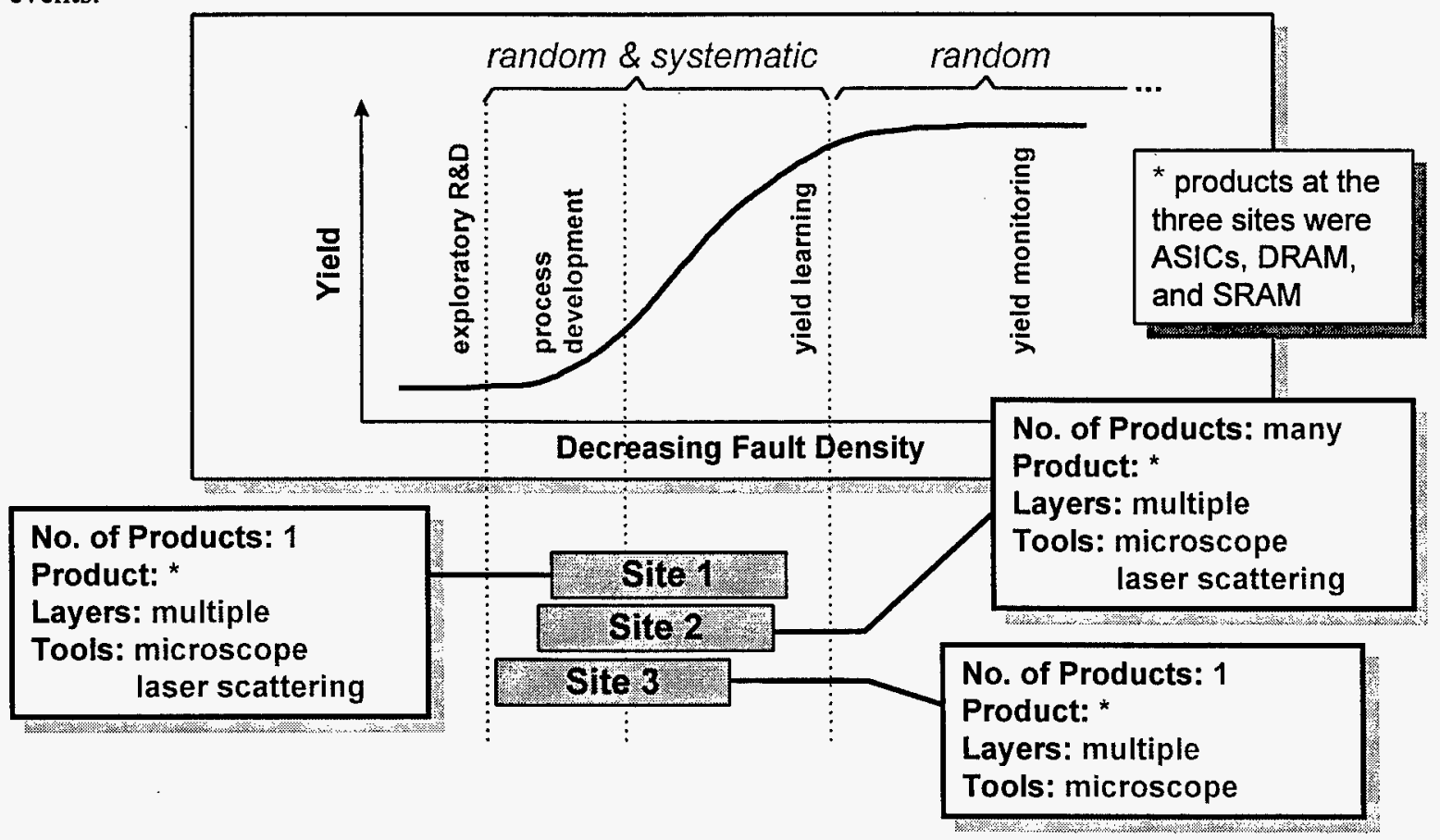

Figure 2. Three sites offered to support the SSA validation activity. These sites produced ASICs, DRAMs, and SRAMs, and represented an early to late process development phase dominated by both random and systematic fault events. 
All three sites selected for the evaluation were operating in the process development / yield learning regions of the curve. At Site 1, SSA monitored one specific product over multiple layers. The wafermap data was generated using in-line microscopy and laser scattering inspection tools. At Site 2, there were several products which went through the in-line microscopy and laser scattering inspection points over the course of the exercise along with a wide variety of process layers. Site 3 was limited to one product but also contained many process layers. At Site 3 only in-line microscopy was used to generate wafermap data for SSA. As mentioned above, the manufactured products at the three sites were ASIC, DRAM, and SRAM.

\section{Optical Defect SSA Validation Results}

Based on the methodology described above, segmentation and classifier performance were evaluated for the data collected at the three manufacturing sites. The following section presents an overall summary of the evaluation at each site. Table 1 gives a summary of classifier and clustering performance (i.e., segmentation) at the three manufacturing sites for various permutations of the data. The first row in the table gives an average classifier performance for all the data analyzed at each site by SSA. This corresponds to 1,933 signatures on 747 wafers that constituted 198 lots and encompassed 50 process steps distributed across the three sites as shown in Table 2.

\begin{tabular}{|l|c|c|c|}
\hline \multicolumn{4}{|c|}{ Table 1. Summary of classifier and clustering performance for the three sites tested } \\
during the validation
\end{tabular}

\begin{tabular}{|l|c|c|c|c|c|}
\hline $\begin{array}{l}\text { Table 2. } \\
\text { and process steps analyzed at the three manufacturing sites. } \\
\text { and }\end{array}$ \\
\hline SITE 1 & 14 & 926 & 604 & 127 & 23 \\
\hline SITE 2 & 12 & 233 & 69 & 31 & 16 \\
\hline SITE 3 & 14 & 774 & 74 & 40 & 11 \\
\hline Total & 40 & 1933 & 747 & 198 & 50 \\
\hline
\end{tabular}

The second row of Table 1 shows the summary statistics for all dominant signatures found in the data. A dominant signature is the critical or most relevant signature that resides on the wafer. Note the significance of higher classifier performance for this category of the data. Combined with a clustering performance of $100 \%$ for all dominant signatures, this signifies that SSA is very capable of detecting and identifying important wafermap signature events, i.e., those signatures that are most likely to open a window into the manufacturing process and lead to fast process characterization and correction.

The remaining rows of performance data in Table 1 show the performance and clustering capability of SSA for various whole-wafer defectivity levels. It was initially anticipated that classification performance would be highest for low-defectivity levels on wafers (due to the simple morphology of the signature structures) but the data suggests a fairly uniform (at least uncorrelated) distribution of performance across 
the ranges of $0-99,100-999$, and 1,000-20,000 defects per wafer. Clustering performance, on the other hand, does show a trend towards decreased efficacy as the wafer becomes more populated with defects. This is likely a result of confusion caused by an increasing number of high-density, overlapping signatures.

Because of the overall success of the SSA approach to analyzing optical defect wafermaps, there has been interest in applying the same technique to other parametric data gathered from the wafer manufacturing process. Specifically, the application of SSA to electrical test data has generated significant interest in the semiconductor manufacturing community. The following section describes the first extension of SSA to other wafer diagnostic data.

\section{TECHNICAL APPROACH}

Here we present the methodology for performing an SSA-based analysis on electrical test bin data.

\section{Binmap Image Creation}

To perform an analysis on the 2-D distribution of bin data, it is convenient to convert the binmap into an image for subsequent processing. The pixels in the created binmap image correspond to the die on the wafer, and the value of a pixel is set according to the bin classification of the corresponding die. For example, if we are interested in analyzing the pattern of die in the FAIL bin, we would generate an image whose pixels that correspond to FAIL die would have a value of 1 while the rest of the pixels would have a value of zero. If one wishes to analyze a stack of e-test binmaps for several wafers in a lot, then each pixel in the created binmap image is assigned a value equal to the number of wafers whose corresponding die fall into the bin of interest. The resulting binmap image can be thought of as two-dimensional histogram where the $(x, y)$ pixels of the histogram represent the position of a die on the wafer, and the histogram value, $h(x$, $y$ ) is the number of die within the wafer stack that fall into the bin under consideration. Because of the nature of bin data, the resulting binmap image has a resolution that corresponds to the size of the die on the wafer. Fig. 3 shows a couple examples of images created from binmaps.
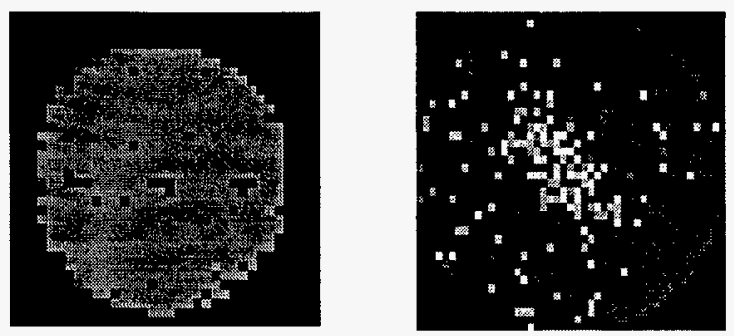

Figure 3. Example binmap images. Gray levels indicate different bin codes and the resolution of the image is equal to the die size.

One of the most notable differences between optical defect wafermaps and binmaps is the resolution of the images created for processing. While the optical defect wafermap resolution is limited only by the resolution of the defect inspection tool, the resolution of the binmap image is limited by the number of die on the wafer (compare Figs. 1 and 3). Because of the limited resolution of binmap images, we have modified (relative to optical defect SSA) the collection of SSA features extracted to describe the binmap signatures. The features used for this analysis are described in the following section.

\section{Feature Extraction}

The features that were chosen for the binmap pattern analysis are for the most part, a subset of the features used in optical defect SSA. Specifically, the features extracted from the binmap images for subsequent 
classification are mass, a set of moments, and a set of invariant moments. For an image whose intensity value at point $x, y$ is $f(x, y)$, the features are calculated as follows:

Mass, $M$ :

The mass of the image is calculated using the following formula:

$$
M=\frac{\sum_{x} \sum_{y} f(x, y)}{N}
$$

where $N$ is the total number of pixels in the image. The mass is normalized so that there will be consistency between binmaps with different numbers of die (or pixels if we are concerned with the binmap-derived image). The mass of the image provides a general idea of how large the distribution of die is within the bin of interest, but does not provide any information about the location of the distribution. Location information is obtained by measuring the centroid of the image.

Centroid, $\left(x_{c}, y_{c}\right)$ :

The centroid of the binmap image is found using:

$$
x_{c}=\frac{\sum_{x} \sum_{y} x \cdot f(x, y)}{\sum_{x} \sum_{y} f(x, y)} \quad y_{c}=\frac{\sum_{x} \sum_{y} y \cdot f(x, y)}{\sum_{x} \sum_{y} f(x, y)}
$$

The centroid gives the location of the center of mass of the distribution of the die, but provides no other information about the extent or shape of the distribution. For this information, moment features are used extensively.

Moments of order $(p, q), m_{p q}$ :

Multiple-order moments of an image can be measured with the following formula:

$$
m_{p q}=\frac{\sum_{x} \sum_{y} x^{p} \cdot y^{q} \cdot f(x, y)}{\sum_{x} \sum_{y} f(x, y)}
$$

In the SSA implementation for binmap analysis, the moments for every pair-wise combination of $p=0, \ldots 3$ and $q=0, \ldots .3$ are calculated and used as features for subsequent classification. An additional set of invariant moments ${ }^{10}$ can be derived from $m_{p q}$ that provide some advantages for this application as described next.

Invariant moments, $\phi_{i}$ :

The invariant moments are features derived from the central moments that can be used as image descriptors. The advantage of these moments is their invariance to image translation, rotation, and scale changes. The formulas for the seven invariant moments are shown below.

$$
\begin{gathered}
\phi_{1}=\mu_{20}+\mu_{02} \\
\phi_{2}=\left(\mu_{20}-\mu_{02}\right)^{2}+4 \mu_{11}^{2} \\
\phi_{3}=\left(\mu_{30}-3 \mu_{12}\right)^{2}+\left(3 \mu_{21}-\mu_{03}\right)^{2}
\end{gathered}
$$




$$
\begin{gathered}
\phi_{4}=\left(\mu_{30}+\mu_{12}\right)^{2}+\left(\mu_{21}+\mu_{03}\right)^{2} \\
\phi_{5}=\left(\mu_{30}-3 \mu_{12}\right)\left(\mu_{30}+\mu_{12}\right)\left[\left(\mu_{30}+\mu_{12}\right)^{2}-3\left(\mu_{21}+\mu_{03}\right)^{2}\right] \\
+\left(3 \mu_{21}-\mu_{03}\right)\left(\mu_{03}+\mu_{21}\right)\left[3\left(\mu_{30}+\mu_{12}\right)^{2}-\left(\mu_{03}+\mu_{21}\right)^{2}\right] \\
\phi_{6}=\left(\mu_{20}-\mu_{02}\right)\left[\left(\mu_{30}+\mu_{12}\right)^{2}-\left(\mu_{21}+\mu_{03}\right)^{2}\right]+4 \mu_{11}\left(\mu_{30}+\mu_{12}\right)\left(\mu_{21}+\mu_{03}\right) \\
\phi_{7}=\left(3 \mu_{21}-\mu_{03}\right)\left(\mu_{30}+\mu_{12}\right)\left[\left(\mu_{30}+\mu_{12}\right)^{2}-3\left(\mu_{21}+\mu_{03}\right)^{2}\right] \\
+\left(3 \mu_{12}-\mu_{30}\right)\left(\mu_{03}+\mu_{21}\right)\left[3\left(\mu_{30}+\mu_{12}\right)^{2}-\left(\mu_{03}+\mu_{21}\right)^{2}\right]
\end{gathered}
$$

Where the $\mu_{p q}$ are the $p, q$ central moments defined as:

$$
\mu_{p q}=\sum \sum\left(x-x_{c}\right)^{p} \cdot\left(y-y_{c}\right)^{q} \cdot f(x, y)
$$

Because the invariant moments can become very large, we took the absolute value of the moments and applied a logarithmic scaling to generate the numbers that are passed on to the classifier as features.

This set of features captures a tremendous amount of information about the distribution of die within the image. As a collection, they provide information about the size, location and shape of the distribution of pixels within the binmap image. In particular, the invariance of some of the features (e.g. $\left.M, \phi_{i}\right)$ is an advantage when one is analyzing binmaps with signature classes that do not depend on scale, location, or angular position.

It is important to note that all of these features will be sent to the fuzzy, pair-wise, k-NN classifier that was developed for previous implementations of SSA for optical defect wafermaps. The classifier will not be discussed in detail here, but the feature-ranking module will be briefly introduced for to make a point. This classifier requires the construction of an exemplar-based library that defines the various classes of binmap signatures, each class containing several examples (e.g. 3-10) of signatures for that class. This library is used to optimally train the classifier to recognize new signatures that fall into the predefined set of classes. Part of the training process is a feature selection algorithm that takes the entire list of aforementioned features and ranks them on a pair-wise class basis. The classifier's ability to rank and select only the features that are pertinent to a class is very important to the success of the entire signature identification system. For example, if a particular binmap signature class contains examples of patterns that have a common shape (e.g. a streak radiating from the center of the wafer), but the pattern can occur at any orientation, it is likely that the rotationally invariant moment features will be ranked relatively high.

\section{PRELIMINARY BINMAP ANALYSIS RESULTS}

A library of binmap signatures has been created, trained, and evaluated. The library contents are summarized in Table 3. Sample maps from some of the classes are shown in Fig. 4. The potential performance of this library was estimated using a hold-one-out technique whereby one signature is held-out of the library, the library was trained on the remaining samples, and the held-out sample was then presented to the classifier. The results of the HOO analysis of the library showed a $94 \%$ correct classification rate. This provides important information about the validity of the classification approach, the represented signature examples in the training library, and, lastly, the discriminatory power of the extracted features. The HOO classification performance measure is not, however, a direct measure of how well the classifier 
will perform on new binmap signatures that are presented to the system. This performance measure is presented next.

\begin{tabular}{|l|l|l|}
\hline \multicolumn{1}{|c|}{ Table 3. } & Binmap signature library description. \\
\hline Class Name & Description & \# Samples \\
\hline good_die & Die that passed all e-test & 4 \\
\hline single & $\begin{array}{l}\text { Single bad die on wafer at any } \\
\text { location }\end{array}$ & 4 \\
\hline double & Two bad die on wafer at any location & 4 \\
\hline low & Few (<20) bad die w/ uniform scatter & 4 \\
\hline linear & 3-5 linearly oriented bad die & 7 \\
\hline heavy & $\begin{array}{l}\text { Heavy distribution of bad die w/ } \\
\text { uniform scatter }\end{array}$ & 4 \\
\hline blob & Concentrated collection of bad die & 4 \\
\hline ring & Die failures along edge of wafer & 6 \\
\hline ring_dash & Ring plus dash-pattern in wafer center & 5 \\
\hline partial_ring & Broken-ring pattern at edge of wafer & 5 \\
\hline
\end{tabular}

We have run approximately 2000 binmaps through the SSA e-test system trained with the previously described signature library and are pleased with the initial performance of the system. It should be noted that the 2000 maps used for testing came from three separate manufacturing sources and from multiple products within each manufacturer. In a true manufacturing environment, the e-test signature library will be much more focused on a particular manufacturer and product, and the performance of this system would be expected to improve. In order to obtain an estimate of the classification performance of the current SSA e-test algorithms, 200 maps were randomly sampled from the current set of 2000 maps and presented to the system. The correct classification rate was $84 \%$ on these randomly sampled maps, which is remarkable

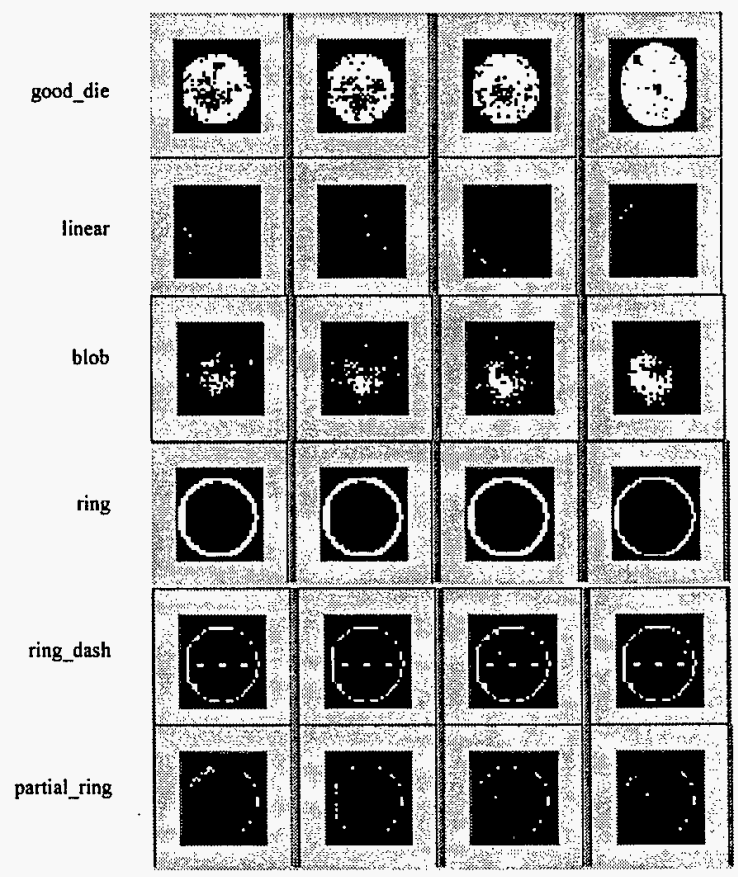

Figure 4. Examples of binmap images stored in the training library. The binmap signature class is given on the left side of each row.

considering the variety of manufacturers and products represented in the training library and in the test 
data. A validation study is now underway at multiple semiconductor manufacturers' sites that will better quantify the performance of the SSA e-test system.

\section{Features of the SSA e-test approach}

There are a few features of the SSA e-test approach to binmap signature analysis that differentiate it from other approaches used to analyze e-test binmaps. One unique feature of the SSA e-test approach is that it is not dependent on the format of the die layout on the tested wafer. Other approaches usually require $a$ priori knowledge of the wafer/die format before the analysis can take place, but the SSA e-test approach is independent of the wafer format because it converts the binmap to an image. It then extracts a complete set of pertinent features from that image, some of which are invariant to image size. This relieves a tremendous burden from the system operator who does not want to spend time reconfiguring their e-test analysis system each time the wafer format is changed. Also, if a system is dependent on the wafer format and the wafer format changes, then all of the previous example binmap data that were used for training become invalid.

A second unique feature of the SSA e-test technique is that the implemented fuzzy classification approach provides training feedback to the operator to inform them of what may be wrong (or right) with their training library. For example, the fuzzy classifier measures the ambiguity on a class-by-class basis during the training process. This information is reported to the operator so that they get immediate feedback if one of their classes is highly ambiguous. They can then proceed to immediately remedy the situation by either adding and/or removing examples from the troublesome class.

\section{CONCLUSION}

An electrical test analysis approach has been developed that converts bin data into an image for subsequent processing and classification. The feature extraction and classification approach for this e-test signature analysis is based on previous work done in the area of optical defect wafermap analysis, namely SSA. The validity of the SSA approach on optical defect wafermaps was demonstrated via an extensive validation study, the results of which were presented. For SSA e-test signature classification, the features extracted from the binmap images include mass, standard moments, and invariant moments. Preliminary testing has been performed by creating an exemplar library, training it, and then classifying 200 randomly sampled binmaps from a collection of 2000 . Even under sub-optimal training conditions, the correct classification rate was $84 \%$. A validation study is underway to more precisely quantify the effectiveness of this approach at multiple semiconductor manufacturing sites.

\section{REFERENCES}

1. K. W. Tobin, S. S. Gleason, T. P. Karnowski, M. H. Bennett, "An Image Paradigm for Semiconductor Defect Data Reduction", SPIE's 1996 International Symposium on Microlithography, Santa Clara Convention Center, Santa Clara, CA, March 10-15, 1996.

2. Gleason, S.S., and Tobin, K.W., "Directional-based Dilation for Connection of Piece-Wise Objects: A Semiconductor Manufacturing Case Study", International Conference on Image Processing, Lausanne, Switzerland, Sept 16-19, 1996.

3. R. O. Duda, and P. E. Hart, Pattern Classification and Scene Analysis, John Wiley \& Sons, New York, 1973, p.95.

4. J. M. Keller, M. R. Gray, and J. A. Givens, Jr., "A Fuzzy K-Nearest Neighbor Algorithm", IEEE Transactions on Systems, Man, and Cybernetics, Vol. SMC-15, No. 4, p. 580, July/August 1985.

5. K. W. Tobin, S. S. Gleason, T. P. Karnowski, S. L. Cohen, "Feature Analysis and Classification of Manufacturing Signatures on Semiconductor Wafers", SPIE's 9th Annual Symposium on Electronic Imaging: Science and Technology, San Jose Convention Center, San Jose, CA, February 9-14, 1997.

6. K. Fukunaga, Introduction to Statistical Pattern Recognition, $2^{\text {nd }}$ Ed., Academic Press, Inc., San Diego, 1990.

7. J. M. Bernardo and A. F. M. Smith, Bayesian Theory, John Wiley \& Sons Limited, West Sussex, England, 1994. 
8. S. Haykin, Neural Networks: A Comprehensive Foundation, Macmillan College Publishing Company, New York, 1994.

9. K.W. Tobin, S.S. Gleason, T.P. Karnowski, S.L. Cohen, and F. Lakhani, "Automatic Classification of Spatial Signatures on Semiconductor Wafermaps", SPIE's 22nd Annual International Symposium on Microlithography, Santa Clara Convention Center, Santa Clara, CA, March 9-14, 1997.

10. R. C. Gonzales, P. Wintz, Digital Image Processing, $2^{\text {nd }}$ ed., Addison Wesley, 1987. 


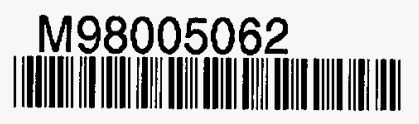

Report Number (14) ORNL/CP-96817

subl. Date (11) $\frac{199802}{D O E / E R, X F}$
$\begin{aligned} & \text { Sponsor Code (18) } \\ & \text { JC Category (19) }\end{aligned}$

\section{0}

